\title{
AVALIAÇÃO DO POTENCIAL DE EXPANSÃO DE ESCÓRIA DE ACIARIA LD: UMA NOVA METODOLOGIA*
}

\author{
Matheus Martini ${ }^{1}$ \\ Fernando Vernilli Júnior ${ }^{2}$ \\ Sidiney Nascimento Silva ${ }^{3}$ \\ André Tarcizo de Oliveira Vieira ${ }^{4}$ \\ Alexander Leal
}

\section{Resumo}

A escória de aciaria LD é um coproduto abudantemente gerado através da metalurgia primária e secundária, sendo que após seu resfriamento e beneficiamento pode ser aplicado em diversos setores, mas que necessita ser submetido a processo de envelhecimento para minimização da expansão. Em geral, no Brasil, o Método PTM-130/78 é o mais utilizado para avaliar o potencial de expansão, entretanto o método leva 14 dias para obtenção dos resultados. Por meio de uma cooperação técnico-econômica entre a CSN e a EEL-USP, o método de expansão foi correlacionado com as fases identificadas e quantificadas por Difratometria de Raios-X e Método de Rietveld, respectivamente, além de compreender o mecanismo de expansão. Análises de regressão múltipla permitiram ajustar modelos matemáticos com $97,0 \%$ de ajuste e uma redução no tempo de ensaio de 14 dias (336 horas) para 7 horas.

Palavras-chave: Método de Rietveld; Escória de aciaria; Expansão de escória; DRX.

\section{EVALUATION OF POTENTIAL FOR EXPANSION OF BOF SLAG: A NEW METHODOLOGY}

\section{Abstract}

BOF slag is an abundant byproduct originated from the hot metal refining to steel and its subsequent types of secondary metallurgy processes. After treatment, BOF slag has many applications, but it needs storage for curing or aging to minimize volumetric instability. In Brazil, PTM-130/78 test is currently the most used to evaluate potential for expansion. However, this method spends 14 days to obtain the result. Through a technical and economic cooperation between CSN and EEL-USP, the traditional method was correlated with the one of X-ray diffraction and the Rietveld Method. Mathematical models obtained by multiple regressions have achieved $98 \%$ of reproducibility and a reduction of 14 days (336 hours) for 7 hours.

Keywords: Rietveld method; BOF slag; Slag expansion; XRD.

1 Engenheiro de Materiais, M.Sc., Assistente Técnico, Departamento de Produto e Assistência Técnica, Togni S/A - Materiais Refratários, Poços de Caldas, MG, Brasil.

2 Engenheiro Químico, Livre Docente, Departamento de Engenharia de Materiais, Escola de Engenharia de Lorena da USP, Lorena, SP, Brasil.

3 Engenheiro Químico, Dr., Gerente de Processos Siderúrgicos, Gerência Geral de Processos Siderúrgicos, CSN, Volta Redonda, RJ, Brasil.

4 Engenheiro Químico, Dr., Coordenador de Gestão de Resíduos, Gerência Geral de Processos Siderúrgicos, CSN, Volta Redonda, RJ, Brasil.

5 Técnico Químico, Supervisor de Planta, Harsco Metals, Volta Redonda, RJ, Brasil. 


\section{INTRODUÇÃO}

$\mathrm{Na}$ indústria siderúrgica há uma grande geração de resíduos, tais como finos e pós, escórias e lamas, mas que podem possuir valor agregado. Dentre os resíduos, a escória de aciaria LD, chamada de agregado siderúrgico após seu beneficiamento, é gerada em grandes volumes (120 kg/t de aço dependendo da siderúrgica) e, em geral, é constituída por uma mistura de silicatos, ferritas combinadas e óxidos livres [1]. Este coproduto pode ser empregado em diversos segmentos, entre eles, na fabricação de artigos de concreto, base e sub-base para pavimento de rodovias, lastro ferroviário, diques marítimos, entre outros. Entretanto, a presença de cal livre $(\mathrm{CaO})$, periclásio $(\mathrm{MgO})$, ferro metálico e a ocorrência do polimorfismo do silicato de cálcio $\left(\mathrm{Ca}_{2} \mathrm{SiO}_{4}\right)$ podem contribuir para a instabilidade volumétrica dos agregados [2-4], sendo necessária a estocagem para envelhecimento ou cura.

Durante a estocagem, o agregado siderúrgico é avaliado sob o ponto de vista do potencial de expansão através da norma PTM130/78 (Pennsylvania Test Method) [5], que estabelece 3\% como limite máximo. O ensaio leva 14 dias o que, muitas vezes, acaba ultrapassando a capacidade do laboratório por conta do acúmulo de material para ensaio, podendo prejudicar a qualidade e a confiabilidade de aplicação dos produtos.

Uma parceria técnico-econômica estabelecida entre EEL-USP e CSN aliou técnicas tradicionais de difratometria de raios X e Método de Rietveld [6-8] para proporcionar soluções tecnológicas e de engenharia que pudessem agregar conhecimento e valor, otimizando os processos e a qualidade dos coprodutos.

\section{MATERIAIS E MÉTODOS}

Foram desenvolvidas atividades experimentais com equipamentos disponíveis na Harsco Metals \& Minerals, na Usina Presidente Vargas - Companhia Siderúrgica Nacional, no Departamento de Engenharia de Materiais da Escola de Engenharia de Lorena, Universidade de São Paulo (DEMAR EEL-USP) bem como no Centro de Previsão de Tempo e Estudos Climáticos (CPTEC) do Instituto Nacional de Pesquisas Espaciais (INPE). Durante a abordagem experimental, foi gerado um lote de escória de aciaria LD, de aproximadamente $300 \mathrm{~m}^{3}$. Após o beneficiamento (britagem, separação magnética e peneiramento), as amostras-teste referentes aos agregados de 1" (25,4 mm) e 3" (passante em 76,2 mm e retido em 50,8 mm) foram homogeneizadas, quarteadas e condicionadas em pilhas Chevron de $3 \mathrm{~m}^{3}$, com o auxílio de caminhão basculante e pá carregadeira.

Os agregados de 3" foram submetidos a análise microestrutural, realizada no DEMAR EEL USP, utilizando um MEV de bancada, marca Hitachi, modelo TM3000, acoplado com um espectrômetro de dispersão de energia (EDS) o qual permitiu a análise composicional da superfície analisada. O objetivo foi verificar as variações microestruturais com o processo de envelhecimento dos agregados.

Os agregados de 1" foram escolhidos para a avaliação do potencial de expansão através da difratometria de raios-X e Método de Rietveld, pois são mais fáceis de enquadrarem-se no ensaio de expansão PTM-130. O tempo de monitoramento do envelhecimento foi baseado em observações históricas da empresa beneficiadora, Harsco Metals and Minerals, que indica 6 meses de envelhecimento como sendo suficiente para atender a especificação de limite de expansão permissível. 


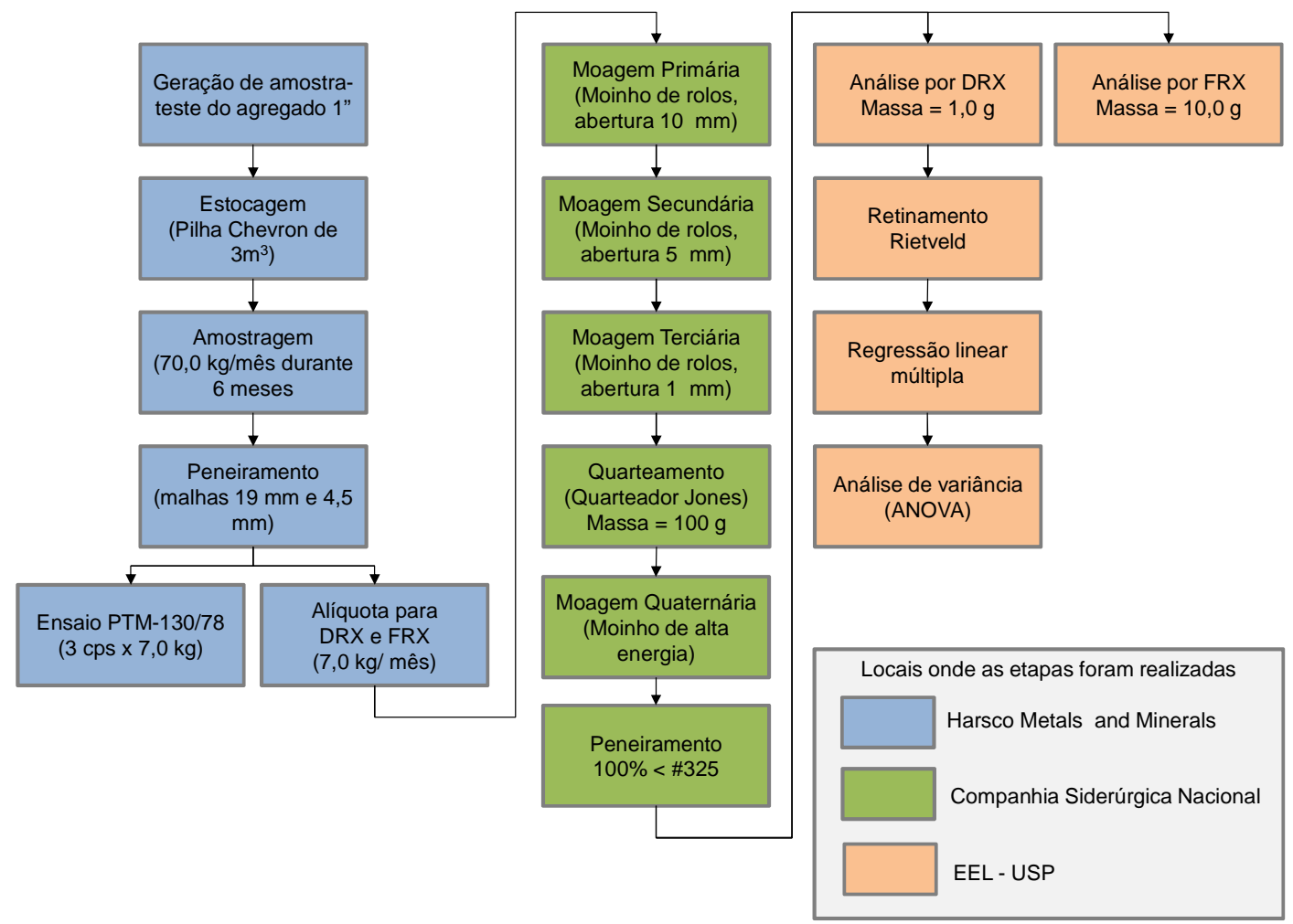

Figura 1. Diagrama geral da metodologia abordada

\subsection{Análise Qualitativa - Difratometria de Raios-X}

As amostras geradas foram analisadas no difratômetro de raios- $X$, marca PANalytical, modelo Empyrean, no DEMAR EEL USP. Em cada mês de envelhecimento obteve-se triplicatas, de aproximadamente $1 \mathrm{~g}$ por quarteamento de 180 gramas da amostra previamente moídas. Adotou-se o Método do Padrão Interno, que consistiu na adição de $20 \%$ em massa de rutilo $\left(\mathrm{TiO}_{2}\right)$ em cada amostra, permitindo avaliar o grau de cristalinidade da escória. Os difratogramas foram coletados com radiação de CuKa, segundo as condições apresentadas na Tabela 1. Foi realizado um trabalho comparativo utilizando dois bancos de dados, ICSD e COD $[9,10]$, no intuito de verificar a convergência de fases identificadas nas amostras analisadas.

Tabela 1. Condições instrumentais utilizadas na realização das análises por DRX

\begin{tabular}{ll}
\hline Parâmetros & Características \\
\hline Radiação & CuKa $(\lambda=1,5460 \AA)$ \\
\hline Tubo & Foco largo (BF) \\
\hline Ângulo de take off & $6^{\circ}$ \\
\hline Voltagem no tubo & $40 \mathrm{kV}$ \\
\hline Corrente no tubo & $30 \mathrm{~mA}$ \\
\hline Fenda Soller & $0,2 \mathrm{~mm}$ \\
\hline Fenda de divergência & $14^{\circ}$ \\
\hline Fenda de recepção & $0,2 \mathrm{~mm}$ \\
\hline Fenda de espalhamento & $1 / 2^{\circ}$ \\
\hline Ângulo inicial $\left({ }^{\circ} 2 \theta\right)$ & 10,0 \\
\hline Ângulo final $\left({ }^{\circ} 2 \theta\right)$ & 90,0 \\
\hline Tempo por passo angular $(\mathrm{s})$ & 100 \\
\hline Passo angular $\left({ }^{\circ} 2 \theta\right)$ & 0,01 \\
\hline
\end{tabular}




\subsection{Análise Quantitativa - Método de Rietveld}

O Método de Rietveld foi realizado utilizando os dois bancos de dados gerando ao total 42 difratogramas, sendo 21 difratogramas utilizando a base de dados ICSD e 21 difratogramas utilizando a base de dados COD. O programa, HighScore Plus PANalytical, além de identificar as fases componentes da escória, permitiu a quantificação destas, que por sua vez, foram correlacionadas com a expansão acumulada medida através do método PTM-130/78. Algumas estratégias para refinamento foram necessárias [11], sendo brevemente exibidas no diagrama da Figura 2.

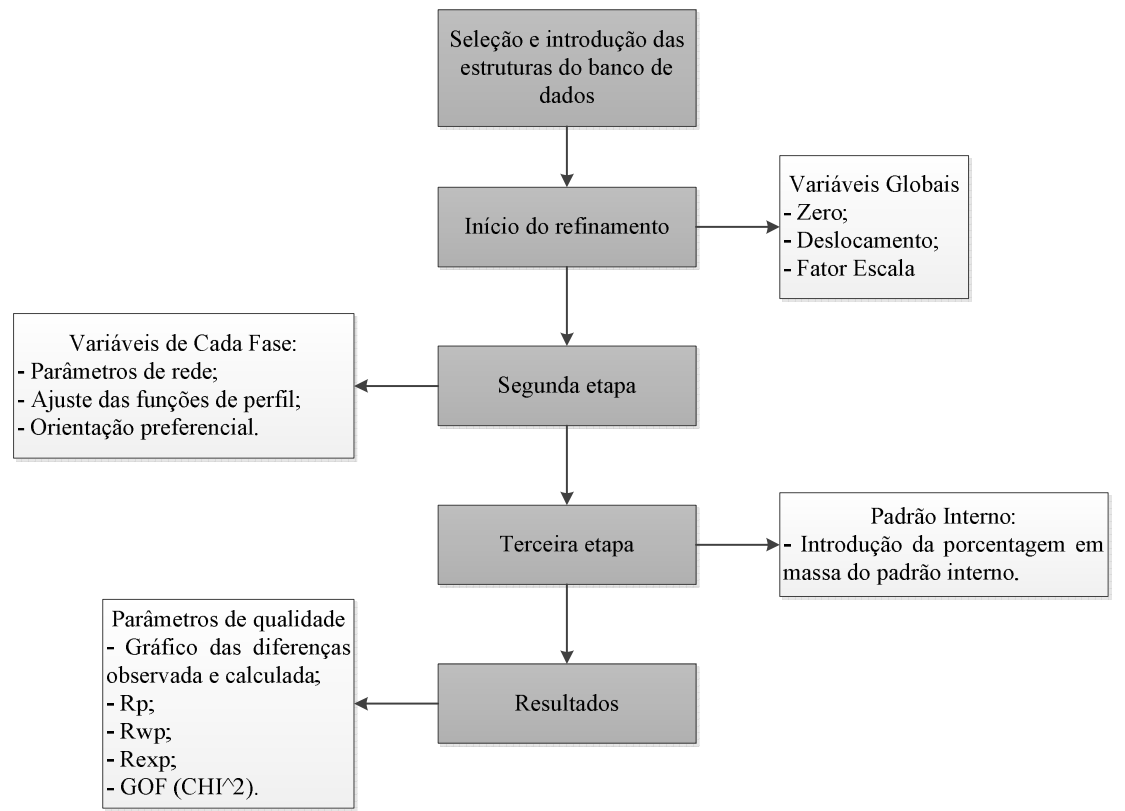

Figura 2. Fluxograma esquemático das etapas de refinamento dos difratogramas.

\subsection{Análise Estatística}

Após as análises de DRX e refinamento Rietveld, utilizando os dois bancos de dados, identificaram-se em torno de 10 fases presentes nas amostras. Com isso, houve uma dificuldade em identificar e correlacionar as principais fases contribuintes para a expansão no ensaio PTM-130. Foram realizadas regressões múltiplas utilizando o programa Minitab 6.0, uma vez que a regressão possibilita encontrar uma correlação razoável entre variáveis por meio de relações empíricas [12].

Os modelos de regressão foram construídos dentro do intervalo de variação estudado, baseados em testes de hipóteses (teste t e nível de confiança de 95\%) que verificaram a significância de cada variável (neste caso os teores das fases) sobre a expansão. Como utilizaram-se dois bancos de dados no método de Rietveld, foi obtido um modelo de regressão mais ajustado referente a cada banco de dados (ICSD e COD). Cada modelo foi submetido a análises de variância, ANOVA, considerando a situações:

- O modelo obtido por regressão múltipla, utilizando os dados refinados com o COD não difere significativamente da expansão no $14^{\circ}$ dia com o valor medido no ensaio PTM-130;

- O modelo obtido por regressão múltipla, utilizando os dados refinados com o ICSD, não difere significativamente da expansão no $14^{\circ}$ dia com 0 valor medido no ensaio PTM-130. 


\section{RESULTADOS E DISCUSSÃO}

\subsection{Composição Química por FRX}

Através da Tabela 2 os elementos majoritários na escória são: $\mathrm{Ca}, \mathrm{Fe}, \mathrm{Si}, \mathrm{Mn}, \mathrm{Mg}$ e Al. A perda ao fogo, PF, aumentou com o tempo de envelhecimento, provavelmente devido formação de fases susceptíveis à decomposição em temperaturas elevadas, tais como hidróxidos e/ou carbonatos.

Tabela 2. Composição química dos agregados siderúrgicos em função do tempo

\begin{tabular}{cccccccc}
\hline \multirow{2}{*}{ Determinação (\%) } & \multicolumn{7}{c}{ Mês de envelhecimento } \\
\cline { 2 - 8 } & $\mathbf{0}$ & $\mathbf{1}$ & $\mathbf{2}$ & $\mathbf{3}$ & $\mathbf{4}$ & $\mathbf{5}$ & $\mathbf{6}$ \\
\hline $\mathrm{Ca}$ & 42,39 & 45,57 & 45,10 & 44,08 & 45,46 & 44,53 & 44,47 \\
\hline $\mathrm{Fe}$ & 30,59 & 30,02 & 32,14 & 31,06 & 29,84 & 30,24 & 29,49 \\
\hline $\mathrm{Si}$ & 8,02 & 7,79 & 8,22 & 8,18 & 8,19 & 7,94 & 7,93 \\
\hline $\mathrm{Mn}$ & 9,44 & 4,44 & 4,42 & 4,39 & 4,36 & 4,68 & 4,43 \\
\hline $\mathrm{Mg}$ & 3,77 & 3,73 & 3,94 & 3,77 & 3,48 & 3,35 & 3,29 \\
\hline $\mathrm{Al}$ & 2,01 & 2,14 & 2,30 & 2,36 & 2,20 & 2,34 & 2,23 \\
\hline $\mathrm{P}$ & 0,79 & 0,73 & 0,76 & 0,79 & 0,79 & 0,76 & 0,75 \\
\hline $\mathrm{Ti}$ & 0,45 & 0,50 & 0,50 & 0,49 & 0,50 & 0,47 & 0,46 \\
\hline $\mathrm{S}$ & 0,18 & 0,21 & 0,16 & 0,20 & 0,21 & 0,20 & 0,19 \\
\hline $\mathrm{Cr}$ & 0,11 & 0,21 & 0,14 & 0,14 & 0,14 & 0,14 & 0,15 \\
\hline $\mathrm{Sr}$ & 0,11 & 0,13 & 0,12 & 0,12 & 0,12 & 0,12 & 0,12 \\
\hline $\mathrm{V}$ & 0,07 & 0,07 & 0,07 & 0,07 & 0,07 & 0,09 & 0,08 \\
\hline $\mathrm{Na}$ & 0,06 & 0,09 & 0,05 & 0,06 & 0,05 & 0,05 & 0,06 \\
\hline $\mathrm{Nb}$ & 0,02 & 0,05 & 0,04 & 0,03 & 0,02 & 0,04 & 0,04 \\
\hline $\mathrm{Cu}$ & - & - & - & 0,02 & - & - & - \\
\hline $\mathrm{K}$ & 0,21 & 0,03 & 0,02 & 0,02 & 0,21 & 0,02 & 0,23 \\
\hline $\mathrm{Cl}$ & 0,02 & 0,03 & 0,02 & 0,02 & 0,02 & 0,02 & 0,02 \\
\hline $\mathrm{Zn}$ & 0,02 & 0,02 & 0,02 & 0,02 & 0,02 & 0,02 & 0,02 \\
\hline $\mathrm{PF}$ & 1,76 & 4,24 & 1,96 & 4,20 & 4,34 & 4,99 & 6,05 \\
\hline
\end{tabular}

Nota: (-) abaixo do limite de detecção.

\subsection{Curvas de Expansão}

Através do diagrama da Figura 3, verifica-se que, durante o ensaio PTM-130, ocorre um gradativo aumento da expansão acumulada com o tempo de envelhecimento. Isto é devido a reação do material e a formação de fases que se expandem. Para menores tempos mais pronunciada foi a expansão, sendo que o efeito oposto ocorre para maiores tempos de envelhecimento. Isto é devido à formação de fases que tornam o agregado menos susceptível a se expandir, tais como o $\mathrm{Ca}(\mathrm{OH})_{2}$ e $\mathrm{CaCO}_{3}$, fases estas identificadas por DRX. No entanto, verificou-se que durante o terceiro, quarto e sexto meses ocorreu um aumento da expansão o qual pode estar associado a decomposição do $\beta-\mathrm{CaSiO}_{4}$. 


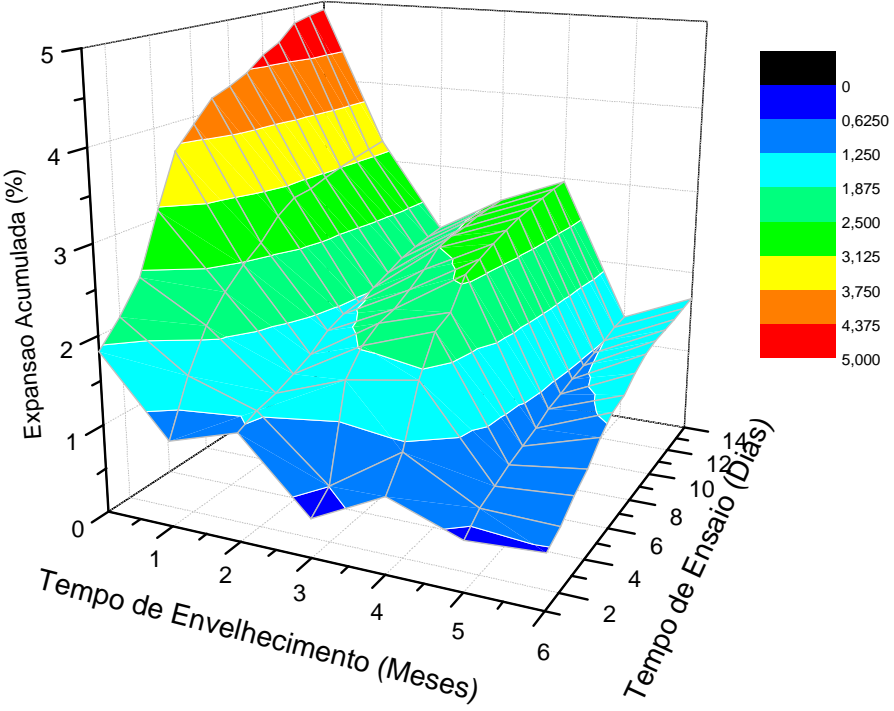

Figura 3. Evolução do potencial de expansão dos agregados siderúrgicos em função do tempo de envelhecimento.

\subsection{Análise de Fases por DRX}

$\mathrm{Na}$ Figura 4 estão exibidos os difratogramas bem como duas regiões que foram ampliadas. As fases identificadas estão apresentadas na Tabela 3. Em geral, observa-se que os perfis dos difratogramas são semelhantes entre si, ou seja, ao longo do tempo de envelhecimento as fases identificadas foram as mesmas, porém variando em sua intensidade. As fases indicadas pelas bases de dados, ICSD e $\mathrm{COD}$, são as mesmas, excetuando-se a fase identificada como número 3 . A base de dados ICSD indicou como sendo uma ferrita de cálcio de relação estequiométrica, enquanto que a base COD indicou uma solução sólida com íons de alumínio substitucional ao íon ferro como sendo a fase mais provável. O pico de maior intensidade refere-se ao padrão interno, rutilo, utilizado para o cálculo do teor de amorfo contido em cada amostra. Constatou-se que, entre os ângulos de $32^{\circ}$ e $38^{\circ}$, há presença de um halo amorfo conjuntamente com picos de calcita, portlandita, ferrita de cálcio, larnita, cal e ferrita de magnésio.

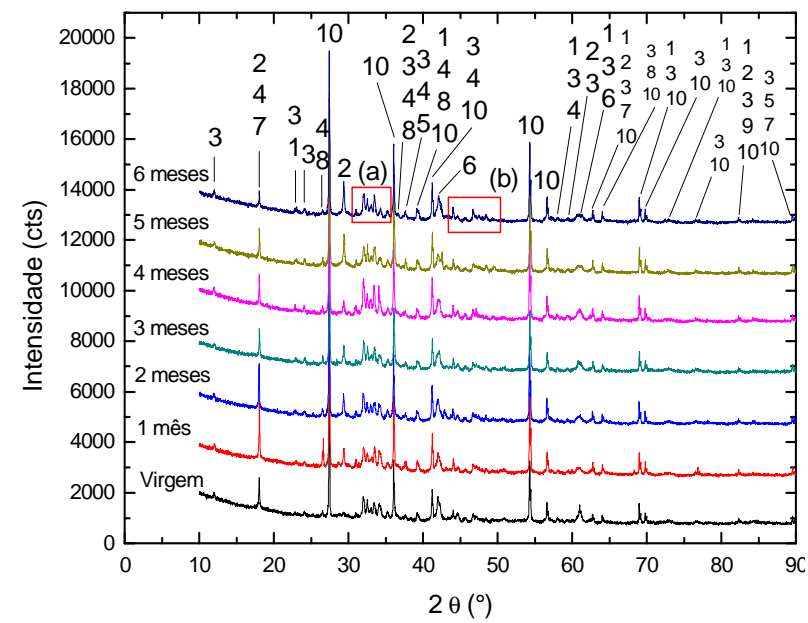

(a)

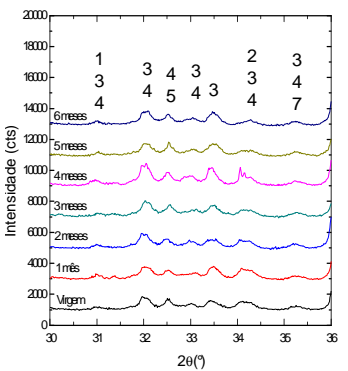

(b)

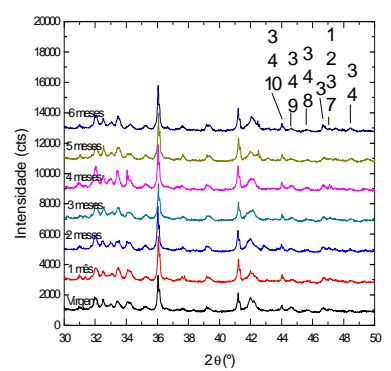

Figura 4. Difratogramas típicos dos agregados siderúrgicos em função do tempo e respectivas regiões demarcadas. 
Tabela 3. Fases componentes da escória de aciaria da CSN.

\begin{tabular}{lllrr}
\hline ID & Fase & Fórmula Química & Código ICSD & Código COD \\
\hline 1 & Calcita & $\mathrm{CaCO}_{3}$ & 20179 & $96-100-0045$ \\
\hline 2 & Portlandita & $\mathrm{Ca}(\mathrm{OH})_{2}$ & 15471 & $96-900-0114$ \\
\hline 3 & Srebrodolskita/ Brownmillerita & $\mathrm{Ca}_{2} \mathrm{Fe}_{2} \mathrm{O}_{5} / \mathrm{Ca}_{2} \mathrm{Fe}_{1,56} \mathrm{Al}_{0,44} \mathrm{O}_{5}$ & 14296 & $96-900-3343$ \\
\hline 4 & Larnita & $\beta-\mathrm{Ca}_{2} \mathrm{SiO}_{4}$ & 81097 & $96-901-2790$ \\
\hline 5 & Cal & $\mathrm{CaO}$ & 26959 & $96-100-0045$ \\
\hline 6 & Wustita & $\mathrm{Fe}_{0,957} \mathrm{O}$ & 27851 & $96-101-1169$ \\
\hline 7 & Magnésio-ferrita & $\mathrm{Mg}_{1,5} \mathrm{Fe}_{1,6} \mathrm{O}_{4}$ & 67847 & $96-100-6064$ \\
\hline 8 & Quartzo Baixo & $\mathrm{SiO}_{2}$ & 200721 & $96-901-2602$ \\
\hline 9 & Ferro & $\mathrm{Fe}^{*}$ & 76747 & $96-901-3473$ \\
\hline 10 & Rutilo* & $\mathrm{TiO}_{2}$ & 200391 & $96-900-7433$ \\
\hline
\end{tabular}

Nota: *Padrão adicionado para cálculo da cristalinidade da escória.

\subsection{Método de Rietveld}

A Figura 5 exibe um difratograma típico do agregado siderúrgico ("Iobs") analisado, com seu perfil calculado pelo refinamento Rietveld ("Icalc"), a linha base ("Iback") considerada para o cálculo de teor de amorfo e a diferença entre as intensidades calculadas e as intensidades medidas ("Difference Plot"). Na Figura 6 (a) e (b) estão exibidos os parâmetros de qualidade de ajuste do refinamento dos demais difratogramas. Em geral, para bons resultados $\mathrm{Rwp}<4,0$, sendo que abaixo de 5 são aceitáveis; GoF <2,0, sendo que 1,0 representa um refinamento perfeito, não sendo matematicamente verdadeiro valor menor do que 1,0. Sendo assim, os parâmetros de qualidade indicam que as fases identificadas e os refinamentos matemáticos foram satisfatóriamente ajustados aos difratogramas medidos, permitindo calular os teores das fases na escória.

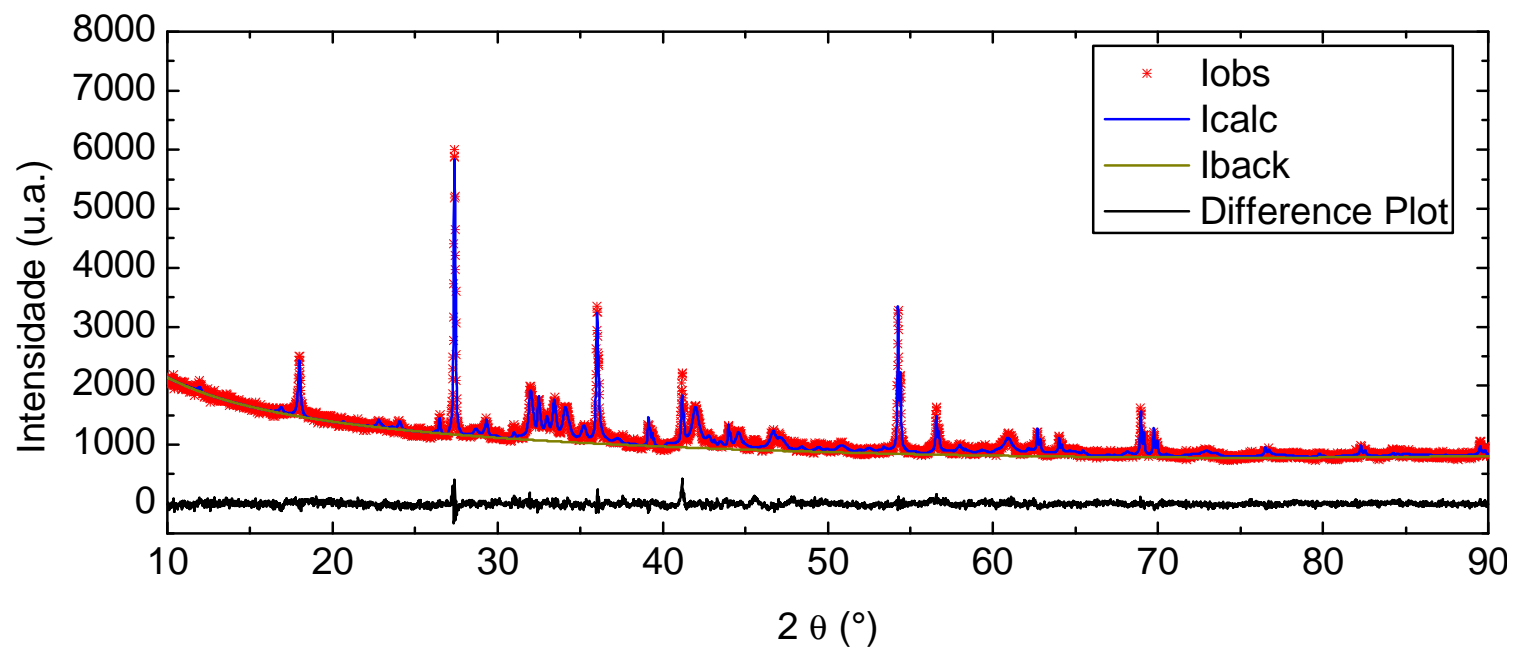

Figura 5. Difratograma típico após a realização do refinamento Rietveld. 


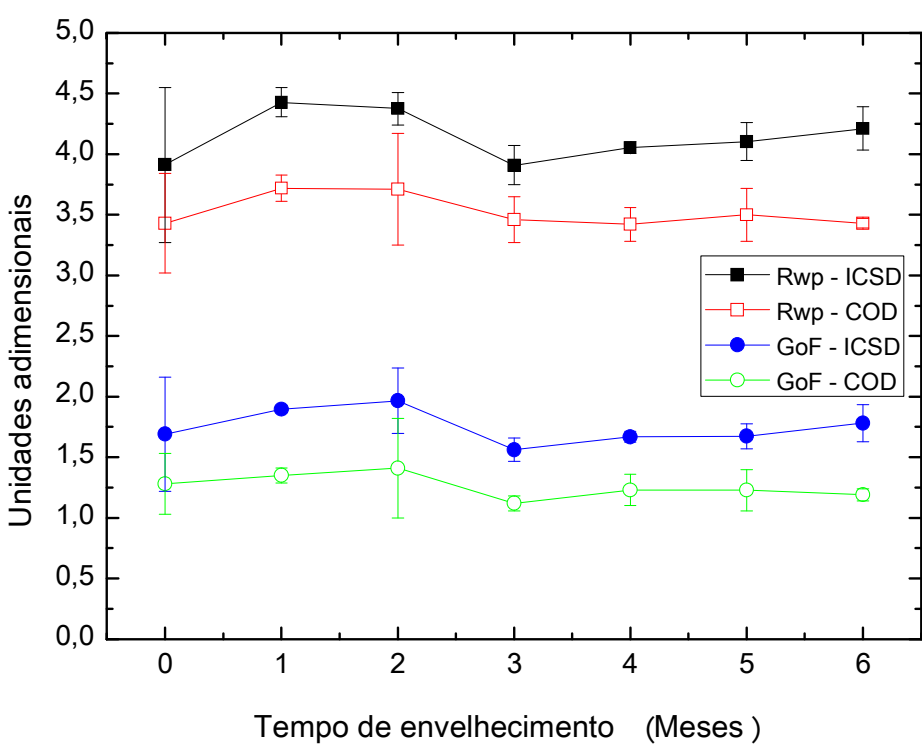

Figura 6. Parâmetros de qualidade do refinamento das fases da escória de aciaria.

\subsection{Análise Estatística: Regressão Linear Múltipla e Anova}

$\mathrm{Na}$ Tabela 4 estão expressos os modelos de regressão múltipla com os melhores coeficientes de ajuste obtidos. Eles foram selecionados dentre os diversos modelos elaborados através dos resultados proporcionados pelo refinamento Rietveld utilizando a base de dados ICSD e COD.

Comparativamente nota-se que o modelo E14-COD não se ajustou, pois apresentou a maior variância e o menor coeficiente de ajuste. Os modelos indicam a influência do óxido de cálcio livre, da portlandita e da calcita na estabilidade volumétrica do agregado siderúrgico. Ainda, com um ajuste satisfatório, pode-se considerar um terceiro modelo contendo a larnita como outra variável. As análises realizadas neste trabalho não permitiram elucidar como esta fase mineralógica age no comportamento expansivo, porém há evidências na literatura[13].

Estatiscamente com 95\% de confiança os modelos E14-ICSD e E14-ICSD' não diferem dos resultados obtidos do ensaio PTM-130/78, pois $\mathrm{P}$-valor $<0,05$; diferentemente do modelo E14-COD que apresentou P-valor >0,05.

Os modelos obtidos e a curva de expansão acumulada no $14^{\circ}$ dia do ensaio PTM130/78 podem ser comparativamente visualizados no diagrama da Figura 7.

Tabela 4. Modelos obtidos por regressão múltipla e indicadores estastísticos

\begin{tabular}{cccc} 
Modelo & $\begin{array}{c}\text { Variância } \\
\mathbf{S}\end{array}$ & $\begin{array}{c}\text { Ajuste } \\
\mathbf{R}^{\mathbf{2}}(\%)\end{array}$ & $\begin{array}{c}\text { P-valor, } \\
(\mathbf{\alpha}=\mathbf{0}, \mathbf{0 5})\end{array}$ \\
\hline $\begin{array}{c}\mathrm{E} 14-\mathrm{COD}=1,86-\left[0,109 \cdot \mathrm{CaCO}_{3}\right]+ \\
{\left[0,345 \cdot \mathrm{Ca}(\mathrm{OH})_{2}\right]+[0,024 \cdot \mathrm{CaO}]}\end{array}$ & 0,987 & 19,100 & 0,379 \\
\hline $\begin{array}{c}\mathrm{E} 14-\mathrm{ICSD}=-3,56+\left[0,237 \cdot \mathrm{CaCO}_{3}\right]+ \\
{\left[0,882 \cdot \mathrm{Ca}(\mathrm{OH})_{2}\right]-[0,188 \cdot \mathrm{CaO}]}\end{array}$ & 0,204 & 97,000 & 0,003 \\
\hline $\mathrm{E} 14-\mathrm{ICSD}=-4,21+\left[0,274 \cdot \mathrm{CaCO}_{3}\right]+$ & 0,218 & 96,600 & 0,022 \\
\hline $\left.0,923 \cdot \mathrm{Ca}(\mathrm{OH})_{2}\right]+\left[0,0188 \cdot \mathrm{Ca}_{2} \mathrm{SiO}_{4}\right]-[0,208 \cdot \mathrm{CaO}]$ & & & \\
\hline
\end{tabular}




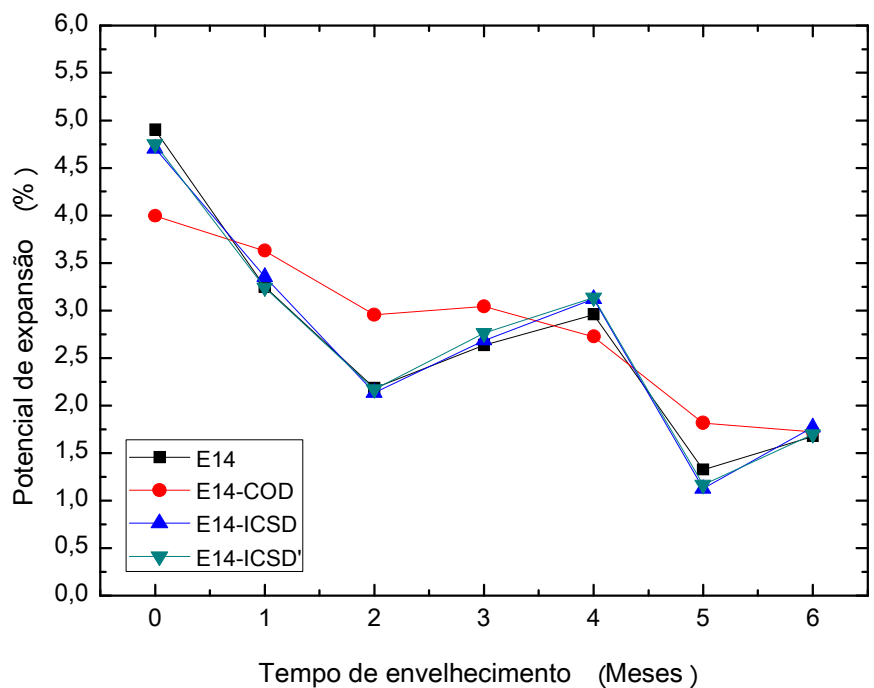

Figura 7. Curvas obtidas da expansão acumulada no $14^{\circ}$ dia do ensaio PTM-130/78 (E14) e dos modelos por regressão linear múltipla. Os pontos foram interconectados e os desvios foram suprimidos para melhor vizualização.

\subsection{Comparação entre os Tempos de Ensaio}

A Figura 8 exibe o tempo médio necessário para realizar cada etapa da metodologia elaborada neste trabalho, bem como o tempo acumulado. A amostragem consiste no quarteamento de $70 \mathrm{~kg}$ até obtenção de $7 \mathrm{~kg}$. A preparação de amostra aborda as etapas de moagem, pesagem e adição de padrão de rutilo. As demais atividades consistem na obtenção do difratograma pela técnica de DRX, a quantificação das fases pelo Método de Rietveld e a elaboração de um relatório técnico dos resultados.

Nota-se que houve uma redução do tempo de ensaio de 336 horas (14 dias) no método tradicional para 7 horas, com resultados estatisticamente indiferentes entre si. Houve, também, um aumento da capacidade de análise, uma vez que tratam-se de amostras com, somente, $1,0 \mathrm{~g}$; diferentemente dos 7,0 kg de cada corpo-deprova introduzido em uma estufa que possui limitações dimensionais sobrecarregando o laboratório.

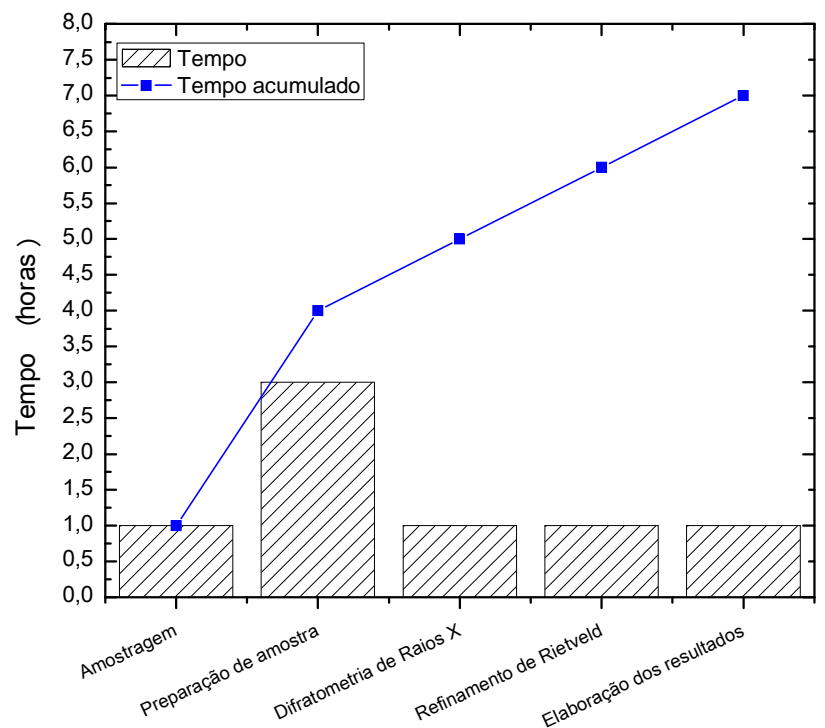

Figura 8. Diagrama ilustrativo do tempo necessário para a realização de cada etapa da nova metodologia desenvolvida. 


\section{CONCLUSÕES}

Neste trabalho, a técnica de difratometria de raios- $X$ indicou que a instabilidade volumétrica das escórias de aciaria é devido, principalmente, à presença dos compostos $\mathrm{CaO}, \mathrm{Ca}(\mathrm{OH})_{2}, \mathrm{CaCO}_{3}$ e $\beta-\mathrm{Ca}_{2} \mathrm{SiO}_{4}$. Além disto, foi identificado que os agregados siderúrgicos podem apresentar propriedades cimentícias, pois foram identificadas as fases $\beta-\mathrm{Ca}_{2} \mathrm{SiO}_{4}$ e $\mathrm{Ca}_{2} \mathrm{Fe}_{1,56} \mathrm{Al}_{0,44} \mathrm{O}_{5}$ nas escórias estudadas.

Através da análise de regressão linear múltipla, utilizando o teor das fases quantificadas pelo método de Rietveld, foi possível elaborar modelos matemáticos bem ajustados, que preveem o comportamento volumétrico dos agregados siderúrgicos sem a necessidade de realizar ensaios de expansão, reduzindo o tempo de análise para 7 horas, bem inferior aos 14 dias (336 horas), necessários para método convencional, PTM130/78.

A difratometria de raios-X e o Método de Rietveld podem ser extendidos a escórias oriundas de outras siderúrgicas, se, e somente se, as devidas metodologias abordadas neste trabalho forem respeitadas, criando-se um modelo estatisticamente adequado para a previsibilidade volumétrica destes materiais.

É importante salientar que a metodologia abordada não é um método absoluto, uma vez que as características físico-químicas dos agregados siderúrgicos são particulares de cada siderúrgica.

\section{REFERÊNCIAS}

1 Machado AT. Estudo comparativo dos métodos de ensaio para avaliação da expansibilidade das escórias de aciaria. [dissertação]. São Paulo (SP): Universidade de São Paulo; 2000.

2 Panis A. Les escories. 1975: 253-258;

3 Kamon M, Montananandh S, Katsumi T. Utilization of stainless-steel slag by cement hardening. Japanese Society of Soil Mechanics and Foundation Engineering. 1993 33(3): 118-129.

4 Heaton BS. Steelworks slag road pavement test sections. Australian Road Research Journal. 1989;19(2): 145-154.

5 Cullity BD. Elements of X-ray diffraction. New York: Addison Wesley Publishing Co., 1978.

$6 \quad$ Klug HP, Alexander LE. X-ray diffraction procedures for polycrystalline and amorphous materials. 2nd ed. New York: John Wiley \& Sons; 1974.

7 Post JE, Bish DL. Rietveld refinement of crystal structures using powder x-ray diffraction data. Mineralogical Society of America. 1989;20: 277-308.

8 Rietveld HM. A profile refinement method for nuclear and magnetic structures. Journal of Applied Crystallography. 1969; 2: 65-71.

9 ICSD, Inorganic Crystal Structure Database [Internet]. Disponível em: http://www.fizkarlsruhe.de/icsd.html.

10 COD, Crystallography Open Database [Internet]. Disponível em: http://www.crystallography.net.

11 McCusker LB, et al. Rietveld refinement guidelines. Journal of Applied Crystallography. 1999; 32: 36-50.

12 Montgomery DC, George CR. Applied statistics and probability for engineers. 3th ed. New York: John Wiley \& Sons; 2003.

13 Kasina M, Michaik M. In: Characterization of convertor slag in terms of slag instability. European Mineralogical Conference; 2012 Sept 2-6; Frankfurt. 